\title{
Is “Frequency Distribution" Enough to Detect Tremor in PD Patients Using a Wrist Worn Accelerometer?
}

\author{
Claas Ahlrichs \\ neusta mobile solutions $\mathrm{GmbH}$ (NMS) \\ Konsul-Smidt-Str. 24 \\ D-28217 Bremen, Germany \\ c.ahlrichs@neusta.de
}

\author{
Albert Samà \\ Technical Research Centre for Dependency \\ Care and Autonomous Living (CETpD) \\ Universitat Politècnica de Catalunya (UPC) \\ Vilanova i la Geltrú (Barcelona), Spain \\ albert.sama@upc.edu
}

\begin{abstract}
This paper presents two approaches on detecting tremor in patients with Parkinson's Disease by means of a wrist-worn accelerometer. Both approaches are evaluated in terms of specificity and sensitivity as well as their applicability for a real-time implementation. One approach is solely based on the frequency distribution of a windowed time series, while the second approach utilizes commonly employed features found in the literature (e.g. FFT, entropy, peak frequency, correlation). The two algorithms detect tremor at rest in windowed time series. The effects of varying window lengths and detection thresholds are studied. The results indicate that an SVM with a linear kernel, in combination with the frequency distribution, may already be enough to accurately and reliably detect tremor in windowed time series. The approach, after being trained with a first dataset of signals obtained from 12 patients, achieved a sensitivity of $88.4 \%$ and specificity of $89.4 \%$ in a second dataset from $64 \mathrm{PD}$ patients.
\end{abstract}

\section{Categories and Subject Descriptors}

J.3 [Computer Applications]: Life and medical science; I.2.6 [Computing Methodologies]: Artificial IntelligenceLearning

\section{General Terms}

Algorithms, Experimentation

\section{Keywords}

Tremor, inertial sensors, Support Vector Machines, learning, accelerometer, Parkinson's Disease

\section{INTRODUCTION}

Parkinson's Disease (PD) is a chronic, progressive, neurodegenerative disorder $[1,12,23]$ that has been first described by James Parkinson in 1817 [16]. It affects the movement

Permission to make digital or hard copies of all or part of this work for personal or classroom use is granted without fee provided that copies are not made or distributed for profit or commercial advantage and that copies bear this notice and the full citation on the first page. To copy otherwise, to republish, to post on servers or to redistribute to lists, requires prior specific permission and/or a fee.

PervasiveHealth 2014, May 20-23, Oldenburg, Germany

Copyright @ 2014 ICST 978-1-63190-011-2

DOI 10.4108/icst.pervasivehealth.2014.254928 of those suffering from the disease and it is typically characterized by a loss of (motor) function, increased slowness and rigidity. Despite radical advancements in medicine and medical technology over the previous two centuries, reason and cause of PD remain unknown [13, 23]. Most treatments aim at reducing severity and frequency of motor complications. They do not cure or have been shown to slow the progression of Parkinson's [13].

Tremor at rest (also known as rest tremor or resting tremor) is an easily (visually) recognized motor symptom of PD. It typically manifests itself as an involuntary, unilateral (onesided) shaking of an extremity (e.g. hand, foot, etc.). In general, extremities of the upper body are more affected then those of the lower body $[14,23]$. The shaking generally occurs at a frequency between $4-6 \mathrm{~Hz}$ [12]. However, various different frequencies can be found in literature [10] (e.g. 4 - 8 $\mathrm{Hz}$ [1] and $3-5 \mathrm{~Hz}$ [23]). This type of tremor is only present when muscles are at rest, hence the name "tremor at rest", and dissolves during sleep as well as with action (i.e. voluntary movement of affected extremity) [14]. Tremor at rest is a common symptom of PD and most people with Parkinson's experience a shaking or trembling in one of their hands at an early stage of the disease [23]. Furthermore, the effectiveness of therapeutic interventions (e.g. levodopa-based medications) can be partially measured through tremor assessment in $\mathrm{PD}$ patients. Thus monitoring of tremor in $\mathrm{PD}$ patients is of main importance and has been widely studied $[2,3,4,8,12,9,18,17,21,24,25]$. The development of small wearable sensor devices largely contributed to the progress in this field.

This paper presents a new method for tremor detection based on learning the spectral distribution of tremor and non-tremor movements. The usage of spectral distribution is compared to the usage of features that have been previously employed to characterize tremor. A literature review on tremor detection through movement sensors is first presented. Then, results obtained for both feature sets are provided based on accelerometer signals obtained from $76 \mathrm{PD}$ patients. Movement signals were taken from the REMPARKproject (Personal Health Device for the Remote and $\mathrm{Au}$ tonomous Management of Parkinson's Disease) database [22]. This project aims at detecting various PD related symptoms in real-time using inertial sensors. Thus, the approach developed in this paper is meant to satisfy a real-time implementation. 
Data collection from PD patients has taken place in 4 different countries. In Spain, Dr. Àngels Bayes led the movement signals gathering from Centro Médico Teknon in Barcelona. Dr. Roberta Annicchiarico coordinated the data collection in Rome, Italy. In Israel, signals were gathered under the coordination of Dr. Hadas Lewy in Maccabi Healthcare Services, Tel Aviv. Finally, signals gathered in Ireland were coordinated by Prof. Gearóid ÓLaighin from National University of Ireland, Galway.

The remainder of this work is structured as follows: Section 2 describes related work with respect to tremor detection in time series, Section 3 provides a general description of the authors' approach to detect tremor, while Section 4 presents detailed information on the data collection and experiments. Section 5 shows the obtained results and, finally, Section 6 summarizes the contributions of this paper and presents future work.

\section{RELATED WORK ON TREMOR DETEC- TION THROUGH INERTIAL SENSORS}

Recently, microelectromechanical systems (MEMS) technology has contributed to develop miniaturized sensors that are capable of continuously monitoring human movement. This way, MEMS technology has opened up new possibilities to study PD and other movement disorders. Given the development of successful machine learning techniques, computer science and artificial intelligence communities are paying more attention to the accurate classification of $\mathrm{PD}$ motor symptoms.

The approaches followed by recent publications may be divided into two phases. First, signals obtained by MEMS sensors are represented by features that are expected to provide a good characterization of tremor presence or absence. Then, the feature-based representation is used as input for a machine learning technique, typically a supervised learning classifier.

In the feature extraction phase, common approaches rely on spectral features $[2,4,8,9,18,21,24,25]$ given the frequency nature of tremor $[10,15]$. Further commonly employed features are Shannon' information features $[4,8,9$, 18], signal entropy [4, 8], as well as first and second-order statistics [8].

Das et al. [8] applied feature extraction to six seconds windows. They determined mean, energy, high frequency energy content, correlation and frequency domain entropy as well as a five bin histogram representation of the spectral contents over all three axes of the accelerometer.

Basu [4] obtained the following measurements from an accelerometer located at the hand in order to characterize tremor: mean frequency, power at peak frequency, mean power in the $j^{\text {th }}$ wavelet band, entropy, sample entropy and mean value obtained of the cell values of a recurrence matrix (following Recurrent Quantification Analysis procedure). The mean recurrence rate was useful in order to quantify the cyclical behavior of the tremor's dynamic system.

Salarian et al. [20] used spectral analysis techniques to find regions within the frequency range of resting tremor (i.e.
$3.5 \mathrm{~Hz}-7.5 \mathrm{~Hz}$ ). In a later study by Salarian et al. [21], the data stream was divided into windows with a length of three seconds to which the Burg method [5] was applied. Additionally a meta-analysis was introduced to remove isolated segments that were classified to exhibit tremor or tremorlike behavior (e.g. a single segment with tremor surrounded by none-tremor segments). This approach resulted in an increased sensitivity but slightly decreased specificity.

Most machine learning techniques used to classify the extracted features as tremor or non-tremor are supervised learning techniques like neural networks (NNs) $[2,3,7,12,19]$ and support vector machines (SVMs) [3, 17]. However, other techniques have also been employed. For instance, Das et al. [8] used an SVM-Multiple Learning approach and Rigas et al. [18] used hidden Markov models to detect tremor episodes.

Finally, tremor has been studied through different sensor locations. A wrist accelerometer is the most common location $[4,9]$ although multiple accelerometers on ankles and waist $[8,18]$ have also been used. In this paper, an accelerometer on the wrist is used as well.

Since REMPARK algorithms are desired to be implemented in an online way, it is necessary to distinguish those approaches that would allow an online implementation on a micro controller from those approaches that do not. Regarding related works, Mutual Information [9] and recurrent matrices are signal features which are not implementable in real-time given the resources needed to compute them. The remaining features, first and second order statistics, entropy and frequency spectra are computable in real-time.

\section{TREMOR DETECTION: PROPOSED AP- PROACHES}

This section describes two complementary tremor detection approaches that will be evaluated on signals obtained from 76 patients.

As described in the related work section, frequency distribution and frequency related features are commonly used to characterize tremor in acceleration signals. The frequency behavior of tremor is illustrated in Figure 1 and Figure 2, which show the frequency distribution of signals obtained from a wrist-worn sensor by a patient. It is clearly observable that frequencies at $4 \mathrm{~Hz}$ to $6 \mathrm{~Hz}$ appear when tremor is present, and these frequencies are not observed when tremor is absent, which agrees with current literature [15]. Given the main frequency behavior of tremor, tremor could be theoretically detected uniquely by means of frequency characteristics. However, many other features have been used in the literature. Thus, in order to measure the impact of nonfrequency features in the accurate detection of tremor, two approaches are defined. On the one hand, the first method will only employ frequency features while, on the other hand, the second approach will also utilize non-frequency features that were previously used in the literature (see Section 2). The specific list of features is given in Table 1.

Following the methods analyzed in Section 2, both approaches will be composed of two-phases in order to determine if tremor is present in a certain time window. The two steps 
Table 1: Commonly utilized features in tremor detection

\begin{tabular}{|c||l|}
\hline Index & Features \\
\hline $001-064$ & FFT (raw, no filtering) \\
\hline $065-066$ & Peak frequency and it's amplitude \\
\hline $067-068$ & Median and mean amplitude \\
\hline $069-084$ & Sum of every two-adjacent amplitudes \\
\hline $085-116$ & Sum of every four-adjacent amplitudes \\
\hline $117-120$ & Sum of first, second and third harmonic \\
\hline $121-136$ & Histogram (bins: $0,1,2,3, \ldots, 15)$ \\
\hline $137-140$ & Correlation between acceleration signal \\
\hline $141-142$ & Entropy of signal \\
\hline
\end{tabular}

are briefly outlined below. The necessary parameter tuning is described in Section 4.

- Feature extraction phase: Features are defined depending on the approach used: frequency features alone or combined with those listed in Table 1. Frequency from three axes must be obtained, and their amplitudes are summed up without taking into account the amplitude of the zero-frequency harmonic. Thus dependence on the sensor's orientation is avoided.

- Learning phase: An SVM is trained to distinguish tremor and non-tremor windows based on the chosen feature set.

Since a real-time implementation of the most successful approach is envisioned, time windows must be short so that a low-consuming microprocessor is capable to analyze its frequency content. Consequently, both methodologies are likely to produce false positives (e.g. a single segment with tremor surrounded by non-tremor segments). These approaches are also likely to produce short non-tremor segments among tremor detections (i.e. while tremor is actually present). Thus, similarly to the approach by Salarian et al. [21], it was decided to add a meta-analysis to enhance the reliability of the proposed approaches by removing isolated segments that were classified to exhibit tremor or tremor-like behavior.

The employed meta-analysis considers the algorithm's outputs in a set of $n$ consecutive windows $s_{1}, \ldots, s_{n}$ that cover a period of $t$ seconds, where $s_{i}=1$ if tremor is present and $s_{i}=0$ if tremor is absent. These outputs are aggregated into a value denoted as $p$ that represents the probability of having tremor in the period of $t$ seconds: $p=\sum \frac{s_{i}}{n}$. Finally, a $t$-seconds period is considered as tremor if the probability $p$ is greater than a certain threshold $0 \leq t h_{p} \leq 1$.

\section{EXPERIMENTS}

\subsection{Data Collection}

In this study, movement signals were recorded for development of a real-time tremor detection algorithm. During data collection, all participants wore a set of body-mounted sensors. In total four sensors in two devices were located on each of the patients. One device was placed around the waist and recorded data from a triaxial accelerometer,

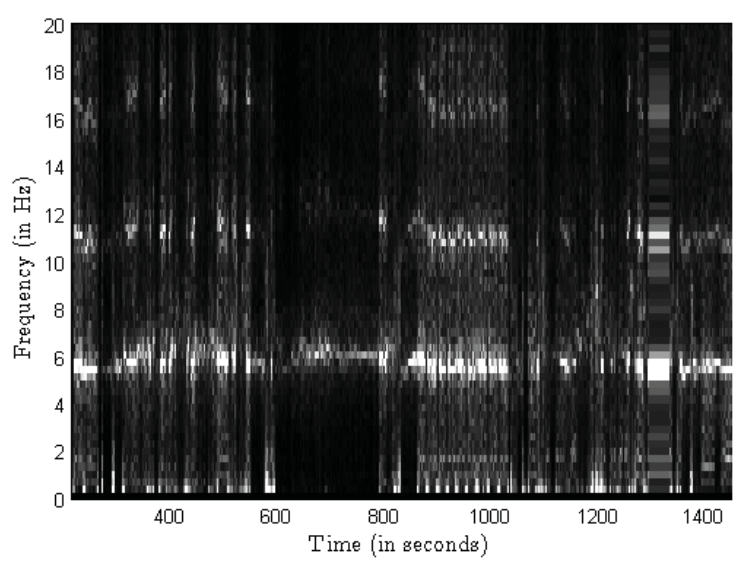

Figure 1: Frequency distribution for individual windows of a patient with tremor. Frequency components around $5-6 \mathrm{~Hz}$ are clearly observable when tremor is present.

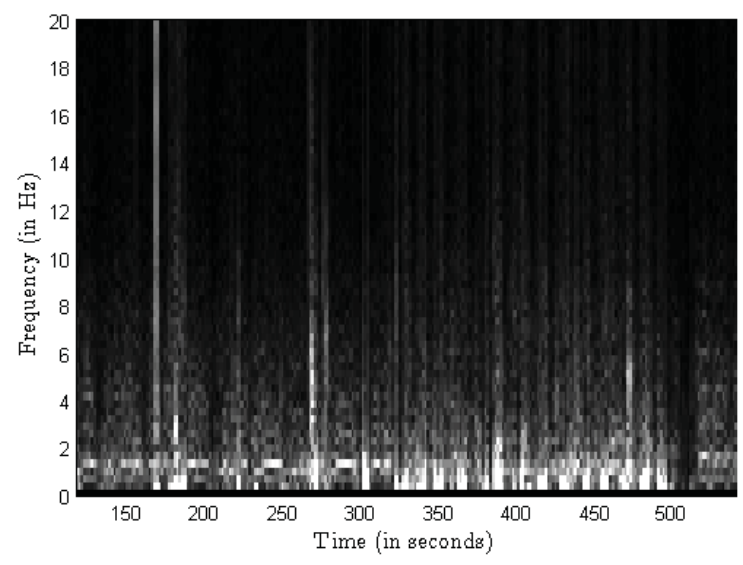

Figure 2: Frequency distribution for individual windows of a patient without tremor.

magnetometer and gyroscope at $200 \mathrm{~Hz}$. The other device recorded data from a triaxial accelerometer worn around the wrist, similarly to a regular watch. Latter sensor transmitted accelerometer data sampled at $80 \mathrm{~Hz}$ to the waist sensor via Bluetooth, where all measurements were stored in a $\mu \mathrm{SD}$ card. In this study, only the wrist device is considered. Once the signals were recorded, a clinician labeled the signals and provided the presence or absence of tremor in each limb.

Participants were video recorded while they performed a set of scripted activities as well as unscripted activities in their own home / apartment (i.e. once in a clinically OFF state and once in a clinically ON state) [22]. Labeling was done by medical professionals using the recordings as reference. Sensor signals and video recordings were synchronized by means of an abrupt translation of the sensor while it was visible in the video recording. Only a subset of the signals was used, namely those after the sensors were successfully placed on the patient and right up to the point where the sensors were taken off. Thus parts of the signal at the begin- 
ning (before initial synchronization point) and ending (after final synchronization point) are removed as they cannot be utilized (and do not contain usable information).

Patients who participated had a clinical diagnosis of Idiopathic Parkinson's disease according to the UK Parkinson's Disease Society Brain Bank [11]. The experimental protocol was approved by the corresponding local Ethics Review Committee and patients gave signed informed consent.

\subsection{Tremor Detection Algorithms}

The time series are resampled to $40 \mathrm{~Hz}$ and divided into equally sized windows, each window having a length of 3.2 seconds (i.e. 128 samples) and a $50 \%$ overlap (i.e. a new window every 64 samples or 1.6 seconds). A set of features (e.g. FFT, peak frequency and amplitude, entropy, etc.) is calculated for each window. A complete set of these features can be reviewed in Table 1 .

Only those windows in which all data samples are labeled as tremor or non-tremor, are utilized for training (i.e. windows containing multiple labels are discarded during training). Furthermore, the selection of windows is restricted to those that do not have missing data (e.g. due to communication problems). This constraint was employed as a mere precaution. An analysis revealed that this was the case in less than $1 \%$ of recorded samples.

Three non-overlapping datasets (i.e. training, holdout and testing dataset) are used to train, optimize and test the tremor detection approaches. An SVM is trained using the training dataset and the libSVM library [6]. The best kernel, weighting, cost and gamma values were determined. The weighting parameters are used to balance tremor and non-tremor classes. The cost and gamma parameters were systematically evaluated (i.e. $0.001,0.01, \ldots, 100,1000$ ) depending on the chosen kernel (i.e. RBF kernel or linear kernel). Their optimal values were found through a stratified ten-fold cross-validation. However, instead of averaging the accuracy of the training set, the maximum geometric mean

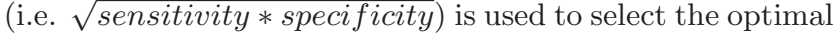
cost and gamma parameters for the final SVM models. The performance of each SVM model is determined by means of the test dataset. However, the restrictions of the training dataset do not apply to these datasets. Patients that do not have any tremor labels or contained only non-tremor labels were automatically relabeled as non-tremor (i.e. the entire patient is relabeled).

In total, four conditions were evaluated: two kernels (i.e. RBF and linear) and two feature sets (i.e. FFT only and FFT + other commonly employed features). Additionally, their performance on varying levels of granularity is evaluated for $t=15,30,45,60$ seconds. The original labeling was automatically refined such that a corresponding (aggregated) label for each aggregated window is determined. If at least one tremor label is among the labels, then the aggregated label is set to tremor as well. Otherwise the aggregated label is set to non-tremor. Values used for $t h_{p}$, which establishes the minimum probability to consider a period of $t$ seconds as tremor, are: $0,0.1, \ldots, 0.9,1$. The SVMs' predicted labels are used to determine the probability of tremor $p$ (see Section 3).
The training dataset is used to train the SVMs in distinguishing tremor and non-tremor frequency distributions / windows. The holdout dataset is utilized to optimize the aggregated window length $t$ as well as the threshold $t h_{p}$ that is required to detect tremor. Finally, the test dataset is used to determine the overall performance of the presented tremor detection algorithm.

\section{RESULTS}

The SVMs' performance on a window-granularity for both approaches can be reviewed in Table 3. The contents are based on the holdout dataset, which contained acceleration signals from $8 \mathrm{PD}$ patients (see Table 2). There, it can be seen that the best performance is achieved using only frequency related features in combination with a RBF kernel. This resulted in $84.6 \%$ sensitivity and $98.0 \%$ specificity. On the other hand, including the rest of features does not seem to affect the overall sensitivity and specificity. Neither seems the use of a linear kernel, as opposed to a RBF kernel, to negatively affect sensitivity and specificity. However, results show that using all features with a linear kernel results in a lower accuracy than only using the frequency features. Since training and holdout datasets do not overlap, this lower specificity implies that learning from nonfrequency distribution features provokes false positive cases. Thus, in the linear kernel case, frequency features seem to provide a better generalization.

Considering the envisioned working environment (i.e. micro controller), it was decided that the "linear kernel with frequency features' case performed accurate enough with no major deviations in terms of sensitivity and specificity (see Table 3). Regardless of the employed kernel (i.e. linear or $\mathrm{RBF}$ ) and number of features (i.e. 64 or 142), the geometric mean measure shows a similar level of performance. Thus the simplest approach was chosen (i.e. using a linear kernel, train frequency distribution and optimization by geometric mean).

Regarding the parameter tuning, the impact of window aggregation $t$ and threshold $t h_{p}$ are measured in Figure 3 in terms of the geometric mean between specificity and sensitivity. A value of zero for $t$ means that no aggregation is done. This case provides the worst case, as no meta-analysis is performed to reduce false positives and false negatives. Optimal performance is obtained for any $t \geq 30$ seconds. According to Figure 3 , the best value for $t h_{p}$ is shown to be among $40 \%$ and $70 \%$, which is reasonably close to $50 \%$, meaning that at least half of the windows in the period of $t$ seconds must be considered as tremor in order to accept the complete period to have the symptom.

The chosen approach (i.e. linear kernel with frequency features with $t=30$ and $t h_{p}=40 \%$ ) results in $88.4 \%$ sensitivity and $89.4 \%$ specificity on the test dataset. More detailed information can be found in Table 4 and Table 5. They show the results of each individual patient in the test dataset. If averaged across all patients (i.e. equal weight for all patients), the sensitivity and specificity result in $85.9 \%$ and $89.3 \%$ respectively. Without the meta-analysis, the sensitivity and specificity further drop down to $84.6 \%$ and $85.6 \%$ respectively (i.e. regardless of whether the patients' number of windows is considered or not). Results for the remaining 


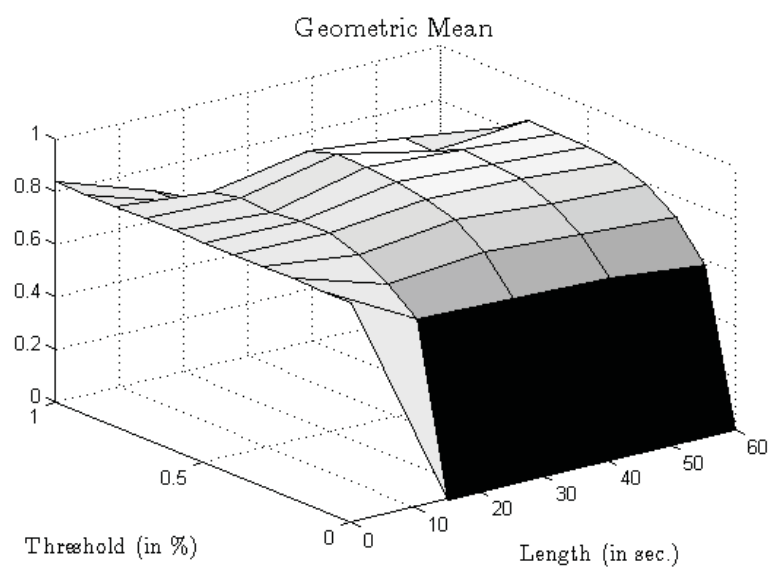

Figure 3: Geometric mean optimization matrix for the 'linear kernel with frequency distribution features' configuration.

Table 2: Number of windows in each dataset. Numbers for the holdout and test dataset correspond to those after automatic relabeling was applied.

\begin{tabular}{|l||c|c|c|}
\hline & Training & Holdout & Test \\
\hline Tremor windows & 717 & 111 & 2339 \\
\hline Non-tremor windows & 1379 & 14645 & 80884 \\
\hline Undefined windows & 3805 & 0 & 0 \\
\hline Discarded windows & 121 & 503 & 11897 \\
\hline Patients in ON state & 3 & 10 & 63 \\
\hline Patients in OFF state & 6 & 6 & 64 \\
\hline Patients (total) & 4.5 & 8 & 63.5 \\
\hline
\end{tabular}

three configurations can be reviewed in Table 6 .

The same configuration yields to a sensitivity and specificity of $89.5 \%$ and $89.7 \%$ (respectively) when the analysis is applied to recordings on OFF state. In ON state, a specificity of $88.9 \%$ was achieved. The sensitivity may be neglected due to its relatively small weight (i.e. 7 samples). Similar results for the remaining three configurations can be found in Table 7 .

Results show that a real-time implementation of the proposed approach is feasible. However, in the experiments, signals obtained while patients did not wear the system, that is, in the beginning and end of the experiments, were rejected. In order to avoid false positives during these situations in a real-time daily use of the proposed approach, a courtesy period of, for instance, 10 minutes after switching on the tremor device could be used. Similarly, the last 10 minutes before switching it off could be rejected.

\section{CONCLUSIONS AND FUTURE WORK}

This paper proposed two different approaches in order to evaluate the impact of using non-frequency features in tremor detection by using a wrist-worn accelerometer. Results on 76 patients show that features obtained from the literature
Table 3: Results for parameter tuning on holdout dataset.

\begin{tabular}{|l||r|r|r|r|}
\hline Kernel & RBF & RBF & Linear & Linear \\
\hline Features & Freq. & All & Freq. & All \\
\hline Features & 64 & 142 & 64 & 142 \\
\hline Length & 30 & 30 & 30 & 45 \\
\hline Threshold & $70.0 \%$ & $70.0 \%$ & $70.0 \%$ & $70 \%$ \\
\hline Sensitivity & $84.6 \%$ & $84.6 \%$ & $84.6 \%$ & $80.0 \%$ \\
\hline Specificity & $98.0 \%$ & $97.0 \%$ & $97.3 \%$ & $97.1 \%$ \\
\hline \# of SVs & 295 & 496 & 428 & 397 \\
\hline
\end{tabular}

Table 4: Results for patients with tremor in the test dataset $\left(t h_{p}=40 \%, t=30\right.$ SECONDS $)$.

\begin{tabular}{|l|r|r|}
\hline Patient & Sens. & Spec. \\
\hline P01 & $100,0 \%$ & $95,6 \%$ \\
\hline P02 & $100,0 \%$ & n/a \\
\hline P03 & $83,3 \%$ & $89,2 \%$ \\
\hline P04 & $75,0 \%$ & $65,0 \%$ \\
\hline P05 & $100,0 \%$ & n/a \\
\hline P06 & $78,6 \%$ & n/a \\
\hline P07 & $64,7 \%$ & n/a \\
\hline
\end{tabular}

Table 5: Results for patients without tremor in the test dataset $\left(t h_{p}=40 \%, t=30\right.$ SECONDS).

\begin{tabular}{|l|r|l|l|l|}
\hline Patient & Spec & & Patient & Spec. \\
\hline P08 & $96,4 \%$ & & P37 & $96,5 \%$ \\
\hline P09 & $100,0 \%$ & & P38 & $98,4 \%$ \\
\hline P10 & $100,0 \%$ & & P39 & $87,3 \%$ \\
\hline P11 & $100,0 \%$ & & P40 & $86,9 \%$ \\
\hline P12 & $100,0 \%$ & & P41 & $83,1 \%$ \\
\hline P13 & $100,0 \%$ & & P42 & $94,2 \%$ \\
\hline P14 & $92,6 \%$ & & P43 & $95,8 \%$ \\
\hline P15 & $100,0 \%$ & & P44 & $99,3 \%$ \\
\hline P16 & $75,0 \%$ & & P45 & $93,4 \%$ \\
\hline P17 & $87,1 \%$ & & P46 & $98,9 \%$ \\
\hline P18 & $92,6 \%$ & & P47 & $95,0 \%$ \\
\hline P19 & $100,0 \%$ & & P48 & $93,3 \%$ \\
\hline P20 & $98,3 \%$ & & P49 & $85,4 \%$ \\
\hline P21 & $98,5 \%$ & & P50 & $85,1 \%$ \\
\hline P22 & $92,4 \%$ & & P51 & $84,7 \%$ \\
\hline P23 & $77,2 \%$ & & P52 & $93,4 \%$ \\
\hline P24 & $82,9 \%$ & & P53 & $83,5 \%$ \\
\hline P25 & $88,9 \%$ & & P54 & $95,5 \%$ \\
\hline P26 & $78,9 \%$ & & P55 & $96,8 \%$ \\
\hline P27 & $94,5 \%$ & & P56 & $71,1 \%$ \\
\hline P28 & $96,7 \%$ & & P57 & $91,2 \%$ \\
\hline P29 & $83,0 \%$ & & P58 & $70,2 \%$ \\
\hline P30 & $90,9 \%$ & & P59 & $79,3 \%$ \\
\hline P31 & $87,7 \%$ & & P60 & $90,4 \%$ \\
\hline P32 & $65,9 \%$ & & P61 & $95,8 \%$ \\
\hline P33 & $59,6 \%$ & & P63 & $93,3 \%$ \\
\hline P34 & $78,0 \%$ & & P64 & $91,8 \%$ \\
\hline P35 & $79,7 \%$ & & P65 & $88,4 \%$ \\
\hline P36 & $96,4 \%$ & & & \\
\hline
\end{tabular}

review, presented in Section 2, do not necessarily increase accuracy (see Table 4. and Table 5. ). Moreover, results 
Table 6: Averaged results for all configurations.

\begin{tabular}{|l||c|c|c||c|c|c|}
\hline \multicolumn{1}{|c||}{} & \multicolumn{2}{c||}{ Without meta-analysis } & \multicolumn{3}{c|}{$\begin{array}{c}\text { With meta-analysis } \\
\left(t h_{p}=40 \%, t=30 \text { sec. }\right)\end{array}$} \\
& Sens. & Spec. & Acc. & Sens. & Spec. & Acc. \\
\hline RBF+Freq. & $84.2 \%$ & $88.0 \%$ & $87.9 \%$ & $92.0 \%$ & $92.0 \%$ & $92.0 \%$ \\
\hline RBF+All & $87.1 \%$ & $84.3 \%$ & $84.3 \%$ & $93.8 \%$ & $88.0 \%$ & $88.1 \%$ \\
\hline Linear+Freq. & $84.6 \%$ & $85.6 \%$ & $85.4 \%$ & $88.4 \%$ & $89.4 \%$ & $89.4 \%$ \\
\hline Linear+All & $86.5 \%$ & $85.5 \%$ & $85.5 \%$ & $92.9 \%$ & $89.7 \%$ & $89.7 \%$ \\
\hline
\end{tabular}

Table 7: Results by motor state for all configurations.

\begin{tabular}{|l||r|r|r||r|r|r|}
\hline \multicolumn{1}{|c||}{} & \multicolumn{3}{c||}{ In OFF state } & \multicolumn{3}{c|}{ In ON state } \\
& Sens. & Spec. & Acc. & Sens. & Spec. & Acc. \\
\hline RBF+Freq. & $92.4 \%$ & $94.5 \%$ & $94.4 \%$ & $85.7 \%$ & $89.9 \%$ & $89.9 \%$ \\
\hline RBF+All & $94.3 \%$ & $90.6 \%$ & $90.8 \%$ & $85.7 \%$ & $85.9 \%$ & $85.9 \%$ \\
\hline Linear+Freq. & $89.5 \%$ & $89.7 \%$ & $89.7 \%$ & $71.4 \%$ & $88.9 \%$ & $88.9 \%$ \\
\hline Linear+All & $93.3 \%$ & $89.2 \%$ & $89.4 \%$ & $85.7 \%$ & $89.9 \%$ & $89.9 \%$ \\
\hline \# of samples & 105 & 1921 & 2026 & 7 & 2474 & 2483 \\
\hline
\end{tabular}

show that a linear kernel may be enough to accurately detect tremor. Thus, it is concluded that frequency features enable reasonably accurate tremor detection. As future work, the approach based on frequency distribution features and an SVM with a linear kernel will be optimized in order to obtain a real-time implementation.

\section{ACKNOWLEDGMENTS}

This work has been performed in the framework of the FP7 project REMPARK ICT-287677, which is funded by the European Community. The authors would like to acknowledge the contributions of their colleagues from REMPARK Consortium (http://www.rempark.eu).

\section{REFERENCES}

[1] R. A. Armstrong. Visual signs and symptoms of parkinson's disease. Clinical and Experimental Optometry, 91(2):129-138, 2008.

[2] R. Arvind, B. Karthik, N. Sriraam, and J. K. Kannan. Automated detection of pd resting tremor using psd with recurrent neural network classifier. In Advances in Recent Technologies in Communication and Computing (ARTCom), 2010 International Conference on, pages $414-417$, oct. 2010.

[3] E. Bakstein, K. Warwick, J. Burgess, O. Stavdahl, and T. Aziz. Features for detection of parkinson's disease tremor from local field potentials of the subthalamic nucleus. In Cybernetic Intelligent Systems (CIS), 2010 IEEE 9th International Conference on, pages $1-6$, sept. 2010.

[4] I. Basu. Design of an ON-OFF closed loop Deep Brain Stimulator for Parkinson's Disease and Essential Tremor. PhD thesis, University of Illinois, 2012.

[5] J. P. Burg. Maximum Entropy Spectral Analysis. PhD thesis, Stanford University, 1975.

[6] C.-C. Chang and C.-J. Lin. LIBSVM: A library for support vector machines. ACM Transactions on Intelligent Systems and Technology, 2:27:1-27:27, 2011. Software available at http://www.csie.ntu.edu.tw/ cjlin/libsvm.

[7] B. T. Cole, S. H. Roy, C. J. De Luca, and S. H.
Nawab. Dynamic neural network detection of tremor and dyskinesia from wearable sensor data. In Engineering in Medicine and Biology Society $(E M B C), 2010$ Annual International Conference of the IEEE, pages 6062-6065, 31 2010-sept. 42010.

[8] S. Das, B. Amoedo, F. De la Torre, and J. Hodgins. Detecting parkinsons' symptoms in uncontrolled home environments: A multiple instance learning approach. In Engineering in Medicine and Biology Society (EMBC), 2012 Annual International Conference of the IEEE, pages 3688-3691, 2012.

[9] M. E. de Oliveira, L. Menegaldo, P. Lucarelli, B. Andrade, and P. Büchler. On the use of information theory for detecting upper limb motor dysfunction: An application to parkinson's disease. Physica A: Statistical Mechanics and its Applications, 390(23-24):4451 - 4458, 2011.

[10] G. Deuschl and C. Goddemeier. Spontaneous and reflex activity of facial muscles in dystonia, parkinson's disease, and in normal subjects. J Neurol Neurosurg Psychiatry, 64(3):320-4, 1998.

[11] A. J. Hughes, S. E. Daniel, L. Kilford, and A. J. Lees. Accuracy of clinical diagnosis of idiopathic parkinson's disease: a clinico-pathological study of 100 cases. Journal of Neurology, Neurosurgery \& Psychiatry, 55(3):181-184, 1992.

[12] J. Jankovic. Parkinson's disease: clinical features and diagnosis. Journal of Neurology, Neurosurgery 86 Psychiatry, 79(4):368-376, 2008.

[13] A. D. Korczyn and T. Gurevich. Parkinson's disease: Before the motor symptoms and beyond. Journal of the Neurological Sciences, 289(1-2):2 - 6, 2010.

[14] A. Krenz. The pathological role of synphilin-1 and the therapeutic potential of Hsp70 in models of Parkinson's disease using viral vectors. $\mathrm{PhD}$ thesis, Universität Tübingen, Wilhelmstr. 32, 72074 Tübingen, 2010.

[15] P. E. O'Suilleabhain and J. Y. Matsumoto. Time-frequency analysis of tremors. Brain, 121(11):2127-2134, 1998.

[16] J. Parkinson. An essay on the shaking palsy. 1817. The Journal of neuropsychiatry and clinical 
neurosciences, 14(2):223-236; discussion 222, 2002.

[17] S. Patel, K. Lorincz, R. Hughes, N. Huggins,

J. Growdon, D. Standaert, M. Akay, J. Dy, M. Welsh, and P. Bonato. Monitoring motor fluctuations in patients with parkinson's disease using wearable sensors. Information Technology in Biomedicine, IEEE Transactions on, 13(6):864-873, nov. 2009.

[18] G. Rigas, A. Tzallas, M. Tsipouras, P. Bougia, E. Tripoliti, D. Baga, D. Fotiadis, S. Tsouli, and S. Konitsiotis. Assessment of tremor activity in the parkinson's disease using a set of wearable sensors. Information Technology in Biomedicine, IEEE Transactions on, $\mathrm{PP}(99): 1,2012$.

[19] S. H. Roy, B. T. Cole, L. D. Gilmore, C. J. De Luca, and S. H. Nawab. Resolving signal complexities for ambulatory monitoring of motor function in parkinson's disease. In Engineering in Medicine and Biology Society,EMBC, 2011 Annual International Conference of the IEEE, pages $4832-4835,30$ 2011-sept. 32011.

[20] A. Salarian, H. Russmann, F. J. G. Vingerhoets, P. R. Burkhard, Y. Blanc, C. Dehollain, and K. Aminian. An ambulatory system to quantify bradykinesia and tremor in parkinson's disease. In Information Technology Applications in Biomedicine, 2003. 4th International IEEE EMBS Special Topic Conference on, pages $35-38$, april 2003.

[21] A. Salarian, H. Russmann, C. Wider, P. R. Burkhard, F. J. G. Vingerhoets, and K. Aminian. Quantification of tremor and bradykinesia in parkinson's disease using a novel ambulatory monitoring system. Biomedical Engineering, IEEE Transactions on, 54(2):313 -322, feb. 2007.

[22] A. Samà, C. Peréz, D. Rodríguez-Martin, J. Cabestany, J. M. Moreno Aróstegui, and A. Rodríguez-Molinero. A heterogeneous database for movement knowledge extraction in parkinson's disease. In European Symposium on Artificial Neural Networks, Computational Intelligence and Machine Learning, 2013.

[23] A. Samii, J. G. Nutt, and B. R. Ransom. Parkinson's disease. The Lancet, 363(9423):1783 - 1793, 2004.

[24] S. L. Smith and K. Shannon. Vector-based analysis of motor activities in patients with parkinson's disease. In EUROMICRO 9\%. 'New Frontiers of Information Technology'. Short Contributions., Proceedings of the 23rd Euromicro Conference, pages 50 -55, sep 1997.

[25] O. Šprdlík, Z. Hurák, M. Hoskovcová, and E. Růžička. Tremor analysis by decomposition of acceleration into gravity and inertial acceleration using inertial measurement unit. In Information Technology and Applications in Biomedicine, 2009. ITAB 2009. 9th International Conference on, pages 1 -4, nov. 2009. 